\title{
Dependence of oxygen desorption kinetics on processing methods of $\mathrm{SrCoO}_{2.5}$
}

\author{
ASWATHY M NARAYANAN* (i) and ARUN M UMARJI \\ Materials Research Centre, Indian Institute of Science, Bengaluru 560012, India \\ *Author for correspondence (aswathyn@iisc.ac.in)
}

MS received 13 June 2020; accepted 17 September 2020

\begin{abstract}
The effect of processing methods on oxygen storage and exchange kinetics of brownmillerite $(\mathrm{BM}) \mathrm{SrCoO}_{2.5}$ is compared in terms of their reversible oxygen incorporation and desorption kinetics. $\mathrm{BM} \mathrm{SrCoO}_{2.5}$ was synthesized through two different methods; solid-state synthesis and the nitrate-citrate gel method, both followed by Al foil pad quenching. Oxygen storage characteristics of synthesized samples were analysed using a home-built volumetric set-up. The maximum oxygenation achievable for $\mathrm{BM} \mathrm{SrCoO} .5$ under prevailing conditions of $\mathrm{O}_{2}$ partial pressure $\left(16\right.$ bar $\left.\mathrm{O}_{2}\right)$, temperature $(673 \mathrm{~K})$ and time $(1 \mathrm{~h})$ was unaltered, regardless of the synthesis method. Oxygen exchange kinetics of desorption was faster in the nitrate-citrate synthesized sample than in the solid-state synthesized sample. The kinetics of desorption correlated with the crystallite and particle sizes of the samples. The mechanism of desorption follows nucleation and growth model for nitrate-citrate synthesized sample, while it follows contracting sphere model for solidstate synthesized sample.
\end{abstract}

Keywords. Oxygen ion diffusion; oxygen non-stoichiometry; mixed ion electron conductor; perovskite oxide; desorption kinetics.

\section{Introduction}

Transition metal oxides have versatile applications in gas sensing, catalysis, cathodes of solid oxide fuel cell and oxygen separation membranes due to the presence of metals with variable oxidation states [1-5]. These metal oxides can have variable oxygen non-stoichiometry with respect to the surrounding temperature and oxygen partial pressure [6-10]. Certain metal oxides even find applications as oxygen sorbents for oxygen storage, separation and enrichment due to its fast response to the changes in ambient oxygen partial pressure and/or temperature [8,9,11-13]. A notable material explored for such applications is brownmillerite (BM) $\mathrm{SrCoO}_{2.5}[11,14,15]$ because of its highest theoretical oxygen intake/release per gram of the material among the other reported oxide materials [11].

Oxygen sorbent transition metal oxide absorbs oxygen from surrounding air atmosphere to fill its lattice oxygen vacancies at a specified temperature range and releases oxygen at a lower oxygen pressure and/or different temperature range $[5,12]$. This necessitates the presence of a large number of oxygen vacancies in the structure, faster oxygen surface exchange and oxide ion diffusion inside the lattice $[5,16]$. Different strategies have been used for tuning these parameters, including aliovalent substitution, forming composites of two materials with one or more of these best properties and using equilibrium/non-equilibrium synthesis Published online: 08 March 2021 methods [17-22]. A large variety of methods have been adopted in literature for the synthesis of mixed oxide ceramics [23-27]. The phase purity, morphology and particle size can be tuned by selecting suitable synthesis methods, which in turn alter the physico-chemical properties $[16,22,24,28]$. The most common methods employed for the synthesis of oxide materials include solid-state reaction [23] and wet chemistry methods like co-precipitation [25], solution combustion synthesis [29] and sol-gel method [24-27].

Conventionally, solid-state reaction (or heat and beat method) between the corresponding metal oxides is widely used for the synthesis of oxide ceramics in large scale because of its simplicity and low manufacturing cost [23]. The relatively high temperatures required to increase solidstate reaction kinetics usually result in low homogeneity, uncontrolled and large particles with a low surface area. To obtain homogeneous materials via solid-state reaction, maximizing the surface area of the reactants so as to improve the reaction rate is considered $[23,24]$. Also, oxysalts of the metals rather than oxides are preferred as precursor materials. These oxysalts are usually decomposed in situ to obtain high surface area oxides, which react further to form the required phase [23]. Another commonly employed method is the sol-gel process, which involves the hydrolysis and polymerization of heterometallic alkoxide precursors to obtain a gel, which is further fired to acquire 
the required oxide [24,27]. The organic part of the alkoxide and $\mathrm{pH}$ conditions are varied for the formation of homogeneous gel, which in turn gives a homogeneous oxide product [27].

Bezdicka et al [30] synthesized $\mathrm{BM} \mathrm{SrCoO}_{2.5}$ using four different methods followed by liquid nitrogen quenching and attempted to oxygenate it by electrochemical technique. They found that the samples differed in their density, particle size and homogeneity with varied synthesis methods. The sample with $80 \%$ density and $1-3 \mu \mathrm{m}$ particle size was found to be optimum for electrochemical oxidation. In this work, the oxygen intake/release properties of $\mathrm{BM} \mathrm{SrCoO}_{2.5}$ synthesized through solid-state reaction and the sol-gel method have been compared. As BM phase is metastable, the phase stabilization was performed by quenching using a heated $\mathrm{Al}$ foil pad for both samples. We further compare the reversible oxygen intake and desorption kinetics of these two samples considering the differences in particle and crystallite sizes.

\section{Materials and methods}

\subsection{Synthesis}

The $\mathrm{BM} \mathrm{SrCoO}_{2.5}$ was synthesized through two different methods.

2.1a Solid-state synthesis: Stoichiometric amounts of $\mathrm{SrCO}_{3}$ (SD Fine Chemicals, 99\%) and $\mathrm{CoC}_{2} \mathrm{O}_{4} \cdot 2 \mathrm{H}_{2} \mathrm{O}$ [31] were weighed, wet mixed with acetone and calcined at $1173 \mathrm{~K}$ for $2 \mathrm{~h}$. The obtained black powder was ground, mixed well and heat treated for $5.5 \mathrm{~h}$ with one intermediate grinding to complete the reaction. Obtained powder was made into a pellet of $10 \mathrm{~mm}$ diameter using a cold uniaxial press. The pellet was soaked at $523 \mathrm{~K}$ for $3 \mathrm{~h}$ to remove the binder and heat-treated at $1223 \mathrm{~K}$ for $6 \mathrm{~h}$ with one intermediate grinding. The pellet was quenched to room temperature from $1223 \mathrm{~K}$ using a pad of heated multilayer Al foil pre-heated at about $373-473 \mathrm{~K}$ to stabilize the intact pellet of high temperature BM phase. The sample obtained through this method is coded as SC-SSS throughout this work.

\section{1b Nitrate-citrate gel method: Stoichiometric} amounts of $\mathrm{SrCO}_{3}, \mathrm{CoC}_{2} \mathrm{O}_{4} \cdot 2 \mathrm{H}_{2} \mathrm{O}$ and citric acid (1:1:3 ratio) were dissolved in hot dilute nitric acid and introduced to $773 \mathrm{~K}$ in a preheated furnace. Water evaporation takes place within few minutes and the formed gel undergoes auto-combustion to give a voluminous black powder. The obtained black powder was mixed well and calcined at 1173 $\mathrm{K}$ for $8 \mathrm{~h}$. It was made into a pellet of $10 \mathrm{~mm}$ diameter using a cold uniaxial press. The pellet was quenched to room temperature as above after sintering at $1223 \mathrm{~K}$ for $4 \mathrm{~h}$. The sample obtained through this method is coded as SC-NC throughout this work.

\subsection{Characterizations}

Phase purity of the samples were analysed using X-ray diffraction (XRD, PANalytical X'Pert Pro, $\mathrm{Cu} \mathrm{K \alpha}$ ). The diffraction pattern was collected in the angular range $10-90^{\circ}$ with a step size of $0.02^{\circ}$ for $20 \mathrm{~min}$ for phase purity analysis. Data collected in the same $2 \theta$ range and step size for $1 \mathrm{~h}$ was used for Rietveld profile refinement to calculate the lattice parameters using GSAS II software [32]. The instrumental profile parameters for the refinement was obtained by refining data of standard $\mathrm{Si}$ sample with minimum crystallite size and strain broadening. The profile shape was calculated using a pseudo-voigt profile function and the irregular background was fitted using manually selected background points and with Chebyshev function.

Morphology of the samples were analysed using a field emission scanning electron microscope (FESEM, FEI INSPECT F50) equipped with an energy dispersive spectrometer (EDS, Oxford Instruments) for elemental analysis. The average particle size of the samples were calculated using Image-J Software [33]. Density of the samples were measured using Archimedes method and was converted to relative density (\% theoretical density) comparing it with that observed from structural data.

Oxygen intake/release studies of the differently synthesized samples were performed in a home-built volumetric apparatus reported elsewhere [14]. In a typical experiment, pieces of the as-synthesized BM sample was pressurized with 16 bar $\mathrm{O}_{2}$ pressure and heated to $648 / 673 \mathrm{~K}$ with a heating rate of $6 \mathrm{~K} \mathrm{~min}^{-1}$. It was soaked at that temperature for $1 \mathrm{~h}$ and cooled back to room temperature in the same oxygen pressure. A known amount of this oxygenated sample was heated to $693 \mathrm{~K}$ with a heating rate of $6 \mathrm{~K} \mathrm{~min}^{-1}$ under reduced oxygen pressure where oxygen desorption takes place. The volume of oxygen which is reversibly incorporated into the material is calculated using the ideal gas equation, $P V=n R T$. Further, isothermal desorption kinetics of the absorbed samples were studied as a function of desorption temperature. Desorption was performed isothermally at 673,693 and $723 \mathrm{~K}$ as a function of time for SC-SSS and SC-NC samples absorbed at $673 \mathrm{~K} / 1$ $\mathrm{h} / 16 \mathrm{O}_{2}$.

Oxygen absorption and release was confirmed by XRD and iodometric titration of as-synthesized, absorbed and desorbed samples. A known amount of the sample $(\sim 30 \mathrm{mg})$ was dissolved in dilute hydrochloric acid containing an excess of potassium iodide. The evolved iodine from the reaction is titrated against standardized sodium thiosulphate solution with freshly prepared starch solution as an indicator towards the end of the titration.

DC electrical conductivities of the samples were measured using four probe Van der Pauw technique [34] on a disc-shaped pellet of $10 \mathrm{~mm}$ diameter and $\approx 2 \mathrm{~mm}$ thickness. Measurements were conducted in the ambient air, flowing nitrogen and flowing oxygen atmospheres from room temperature to $715 \mathrm{~K}$. Electrical contacts were made 
between the sample and platinum leads by applying silver paste (FERRO LAQUE L200).

Linear thermal expansion of as-synthesized and absorbed bar-shaped $\left(10 \times 2 \times 2 \mathrm{~mm}^{3}\right)$ samples were measured using a differential dilatometer (DIL 802, TA Instruments) with respect to alumina reference in the ambient air atmosphere from room temperature to $1000 \mathrm{~K}$.

\section{Results and discussion}

\subsection{Structural characterization}

Figure 1a, b shows the refined XRD patterns of the BM $\mathrm{SrCoO}_{2.5}$ samples, SC-SSS and SC-NC, respectively. Both samples are phase pure and the XRD pattern can be indexed considering a unit cell based on Ima 2 space group [35]. The refined XRD patterns show good fit between measured and calculated profiles. The lattice parameters calculated are given in table 1. Williamson-Hall analysis was carried out to compare the crystallite sizes of the samples obtained through different methods (figure 2). Peaks in the $2 \theta$ range $30-50^{\circ}$ were selected for plotting Williamson-Hall plot. Peaks were de-convoluted assuming Gaussian profile shape. The full-width at half-maxima (FWHM) were corrected by subtracting the FWHM of the standard Si sample $\left(\beta_{\text {actual }}=\right.$ $\left.\left(\beta_{\text {meas }}^{2}-\beta_{\text {inst }}^{2}\right)^{1 / 2}\right)$. The obtained crystallite size and strain values are tabulated in table 1 . The crystallite size of the SC-NC sample was smaller than that of the SC-SSS sample, mainly due to the difference in heat-treatment during the synthesis of these samples (section 2.1).

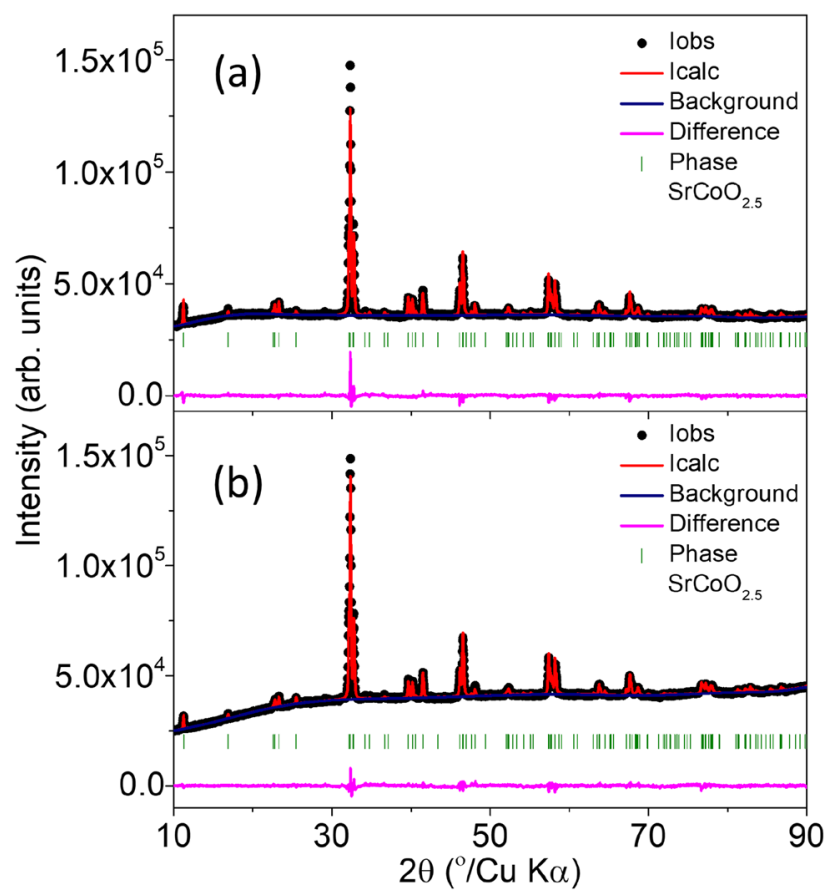

Figure 1. The refined XRD pattern of $\mathrm{BM} \mathrm{SrCoO}_{2.5}$ samples: (a) SC-SSS and (b) SC-NC.

\subsection{Morphological characterization}

SEM micrographs and energy dispersive spectra (EDS) of the SC-SSS and SC-NC samples are given in figure $3 \mathrm{a}-\mathrm{d}$. Both SC-NC and SC-SSS show moderately interconnected globular particles with porous microstructure. The average particle size was slightly different (figure $3 \mathrm{a}$ and $\mathrm{b}$ and table 1), SC-SSS sample had bigger particle size than SC$\mathrm{NC}$ sample. EDS shows the presence of $\mathrm{Sr}, \mathrm{Co}$ and $\mathrm{O}$ in both samples (figure $3 \mathrm{c}$ and $\mathrm{d}$ ).

\subsection{Oxygen storage measurements}

Oxygen absorption and desorption studies were performed with the samples as mentioned in section 2.2. Absorption was conducted with SC-SSS and SC-NC samples at $673 \mathrm{~K}$ by pressurizing with 16 bar of $\mathrm{O}_{2}$ and the desorption was carried out as a function of temperature at a reduced pressure. The XRD patterns of absorbed SC-SSS and SC-NC samples are given in figure $4 a$ and $b$. It can be seen from the figure that both the samples have been converted to perovskite phase. A peak splitting was observed for the peak near $47.4^{\circ}$, indicating a reduced symmetry compared to the true perovskite phase. Thus, I4/ $\mathrm{mmm}$ space group [36] was used to index the XRD pattern of the absorbed samples. Rietveld profile refinement was carried out to obtain lattice parameters and the formula unit cell volumes are tabulated in table 2. Both the samples show a reduction in cell volume compared to the as-synthesized samples indicating oxygen absorption. There is no significant change observed in the normalized cell volume for the two samples, indicating a similar oxygen content. The oxygen content obtained from iodometric titration (table 2) implies the same. Because the as-synthesized samples were found to differ in their crystallite and particle sizes, the reversible oxygen absorption may also differ at a lower temperature. Thus, absorption was carried out at a lower temperature, $648 \mathrm{~K}$. XRD patterns of the absorbed samples (figure $4 \mathrm{c}$ and d) show that it has converted to perovskite phase.

The desorption curves of absorbed SC-SSS and SC-NC samples are given in figure 5. The volume of evolved oxygen starts to increase above a certain temperature as shown in the figure, it reaches a maximum value and remains constant when it is cooled down to room temperature. The total volume of desorbed oxygen was found to be similar in both SC-SSS and SC-NC samples absorbed at $673 \mathrm{~K}$ as well as $648 \mathrm{~K}$ (figure 5 and table 2). The oxygen intake was slightly lower for the samples absorbed at a lower temperature (figure 5 and table 2). The maximum reversible oxygen intake matches with the one reported earlier for a similar sample synthesized through solution combustion synthesis [14]. This reflects the maximum oxygenation achievable for $\mathrm{BM} \mathrm{SrCoO}_{2.5}$ at the absorption conditions to be constant irrespective of the synthesis method employed. XRD patterns of the sample after 
Table 1. Structural parameters of the $\mathrm{BM} \mathrm{SrCoO}_{2.5}$ samples from Rietveld profile refinement, XRD and SEM.

\begin{tabular}{|c|c|c|c|c|c|c|c|c|c|}
\hline \multirow{2}{*}{$\begin{array}{l}\text { Sample } \\
\text { code }\end{array}$} & \multicolumn{3}{|c|}{ Lattice parameters $(\AA)$} & \multirow{2}{*}{$\begin{array}{l}\text { Cell volume } \\
\qquad\left(\AA^{3}\right)\end{array}$} & \multirow{2}{*}{$\begin{array}{l}\text { Cell volume per } \\
\text { formula unit } \\
\qquad\left(\AA^{3}\right)\end{array}$} & \multirow{2}{*}{$\begin{array}{l}\text { Crystallite } \\
\text { size }(\mu \mathrm{m}) \\
(\mathrm{XRD})\end{array}$} & \multirow{2}{*}{$\begin{array}{l}\text { Strain } \\
\text { (XRD) }\end{array}$} & \multirow{2}{*}{$\begin{array}{c}\text { Relative } \\
\text { density } \\
(\%)\end{array}$} & \multirow{2}{*}{$\begin{array}{l}\text { Average particle } \\
\text { size }(\mu \mathrm{m})(\mathrm{SEM})\end{array}$} \\
\hline & $a$ & $b$ & $c$ & & & & & & \\
\hline SC-SSS & $15.726(5)$ & $5.569(2)$ & $5.464(7)$ & $478.526(14)$ & $59.815(2)$ & 0.24 & +0.003 & $85-90$ & $3.0 \pm 0.8$ \\
\hline SC-NC & $15.724(4)$ & $5.568(8)$ & $5.464(8)$ & $478.379(20)$ & $59.797(3)$ & 0.08 & -0.001 & $90-95$ & $2.0 \pm 0.8$ \\
\hline
\end{tabular}

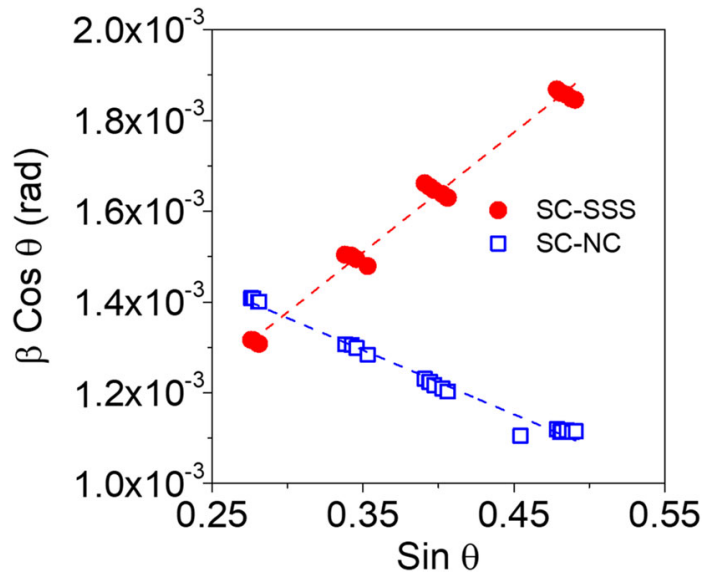

Figure 2. W-H plots for SC-SSS and SC-NC samples. Dashed lines indicate fit to the data points.
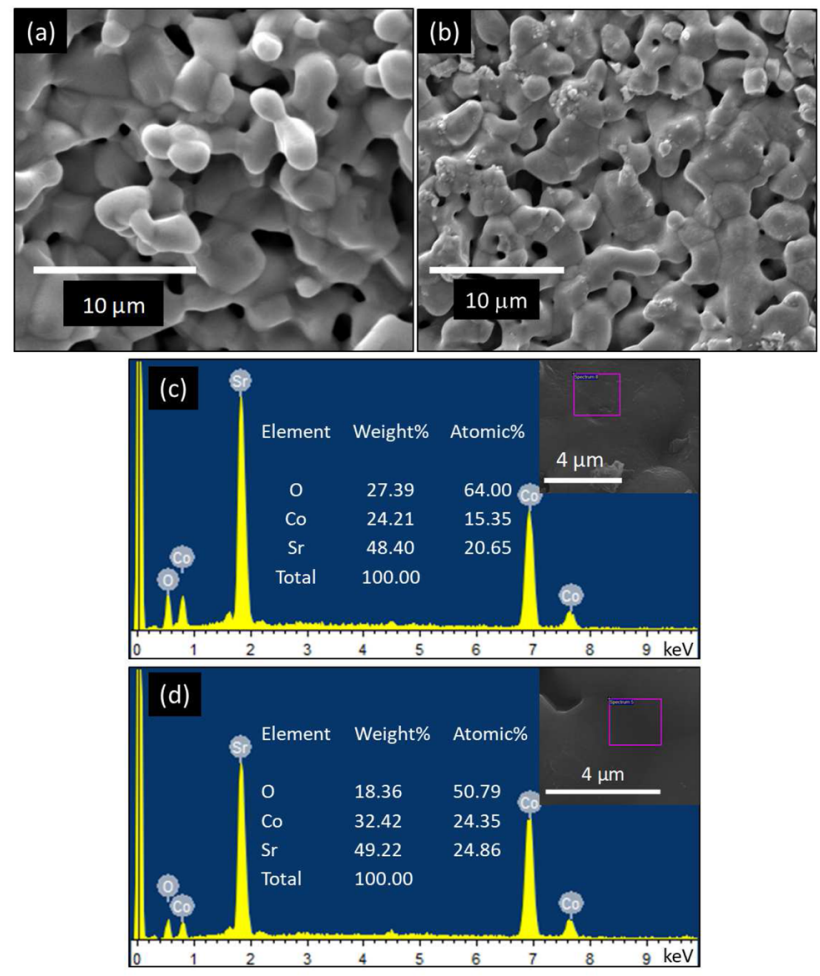

Figure 3. Scanning electron micrographs of $\mathrm{BM} \mathrm{SrCoO}_{2.5}$ samples: (a) SC-SSS and (b) SC-NC. EDS of samples: (c) SCSSS and (d) SC-NC. Elemental composition as obtained from EDS is also shown.

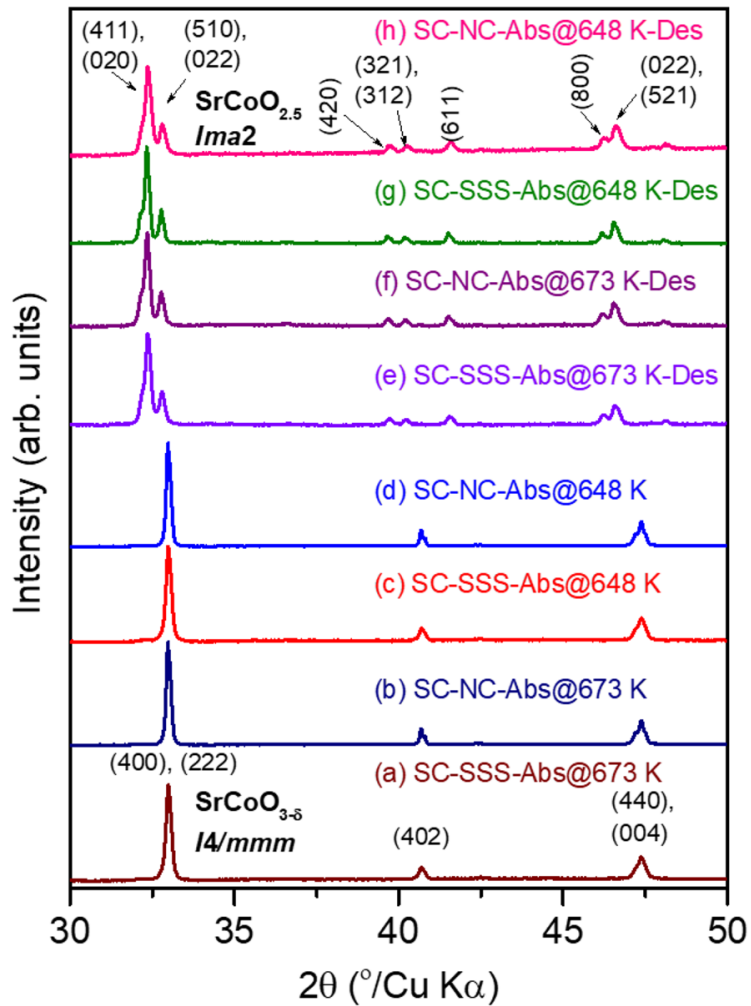

Figure 4. Comparison of XRD patterns of absorbed and desorbed samples: (a) SC-SSS absorbed at $673 \mathrm{~K}$, (b) SC-NC absorbed at $673 \mathrm{~K}$, (c) SC-SSS absorbed at $648 \mathrm{~K}$ and (d) SC-NC absorbed at $648 \mathrm{~K}$. Desorbed after absorption at $673 \mathrm{~K}$ : (e) SC-SSS and (f) SC-NC. Desorbed after absorption at $648 \mathrm{~K}$ : (g) SC-SSS and (h) SC-NC.

desorption indicates a reversible structural change, where the perovskite phase converts back to the BM phase after desorption (figure $4 \mathrm{e}$ and $\mathrm{f}$ ).

\subsection{Kinetics of $\mathrm{O}_{2}$ desorption}

In an oxygen separation/enrichment point of view, an oxygen sorbent will be efficient only if it gives out the absorbed oxygen as fast as possible by an appropriate heat-treatment. The kinetics of oxygen evolution of oxygenated $\mathrm{BM} \mathrm{SrCoO}_{2.5}$ was analysed to understand the effect of synthesis method if any, on oxygen desorption. For this, isothermal desorption of the samples absorbed at 
Table 2. Comparison of oxygen evolved and delta values from desorption experiments and iodometric titration for SC-SSS and SC-NC samples. Cell volume per formula unit of the absorbed samples are also given.

\begin{tabular}{|c|c|c|c|c|c|c|c|}
\hline \multirow[b]{3}{*}{$\begin{array}{l}\text { Sample code and } \\
\text { absorption temperature }(\mathrm{K})\end{array}$} & \multicolumn{2}{|c|}{ Desorption experiment } & \multicolumn{4}{|c|}{ Iodometric titration } & \multirow[b]{3}{*}{$\begin{array}{l}\text { Cell volume per } \\
\text { formula unit }\left(\AA^{3}\right)\end{array}$} \\
\hline & \multirow[b]{2}{*}{$\begin{array}{l}\mathrm{O}_{2} \text { evolved }\left(\mathrm{cm}^{3} \mathrm{~g}^{-1}\right. \\
\left.\text { of } \mathrm{SrCoO}_{2.5+\delta}\right)\end{array}$} & \multirow[b]{2}{*}{$\begin{array}{l}\text { Delta } \\
(\delta)\end{array}$} & \multicolumn{3}{|c|}{ Oxygen content } & \multirow[b]{2}{*}{$\begin{array}{l}\text { Delta } \\
(\delta)\end{array}$} & \\
\hline & & & $\begin{array}{l}\text { As- } \\
\text { synthesized }\end{array}$ & Absorbed & Desorbed & & \\
\hline SC-SSS and $673 \mathrm{~K}$ & $14.5 \pm 0.5$ & 0.24 & $2.38 \pm 0.01$ & $2.67 \pm 0.02$ & $2.46 \pm 0.01$ & 0.21 & $56.490(1)$ \\
\hline SC-NC and $673 \mathrm{~K}$ & $13.5 \pm 0.6$ & 0.22 & $2.43 \pm 0.01$ & $2.69 \pm 0.02$ & $2.43 \pm 0.01$ & 0.26 & $56.571(8)$ \\
\hline SC-SSS and $648 \mathrm{~K}$ & $13.1 \pm 0.5$ & 0.22 & $2.38 \pm 0.01$ & $2.62 \pm 0.01$ & $2.45 \pm 0.01$ & 0.17 & $56.644(7)$ \\
\hline SC-NC and $648 \mathrm{~K}$ & $13.3 \pm 0.3$ & 0.22 & $2.43 \pm 0.01$ & $2.68 \pm 0.02$ & $2.43 \pm 0.01$ & 0.25 & $56.600(2)$ \\
\hline
\end{tabular}

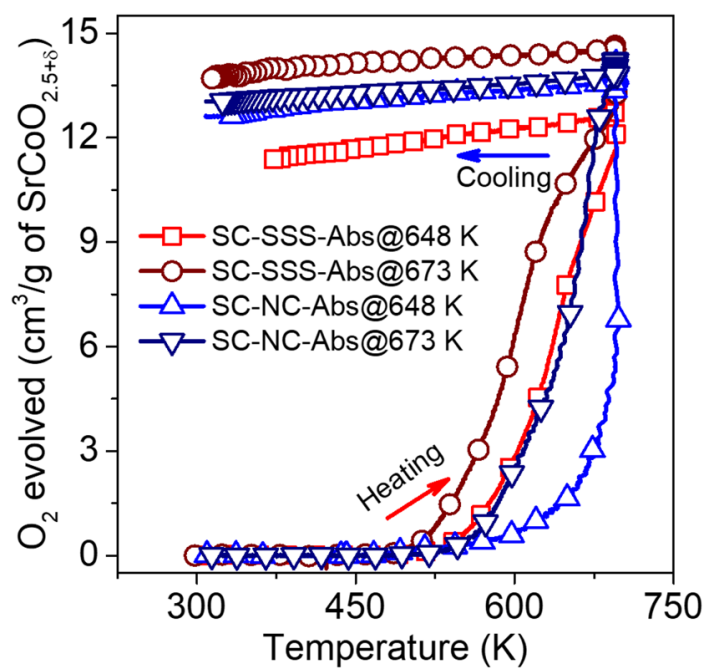

Figure 5. Desorption curves of samples SC-SSS and SC-NC absorbed at 648 and $673 \mathrm{~K}$ for $1 \mathrm{~h}$ in 16 bar $\mathrm{O}_{2}$.

$673 \mathrm{~K} / 16$ bar $\mathrm{O}_{2} / 1 \mathrm{~h}$ was carried out at three different temperatures, 673, 693 and $723 \mathrm{~K}$. The normalized isothermal desorption curves (or the extent of conversion, $\alpha$ ) of SC-SSS and SC-NC samples as a function of time are given in figure $6 a$ and $b$, respectively. Normalization was carried out by dividing the whole curve of evolved oxygen with its maximum value. It can be seen from the figures that the desorption becomes faster for both samples as temperature increases. However, the time scale for desorption was observed to be different for SC-SSS and SC-NC samples (figure $6 \mathrm{a}$ and $\mathrm{b}$ ), SC-SSS samples show a slower desorption compared to SC-NC samples at the same temperature.

The mechanism of reduction of $\mathrm{SrCoO}_{2.5+\delta}$ can be explained using a derivative of the extent of conversion $(\mathrm{d} \alpha /$ $\mathrm{d} t$ ) $v s$. the extent of conversion curve. If $\mathrm{d} \alpha / \mathrm{d} t$ increases and then decreases with an increase in extent of conversion, the mechanism of desorption follows nucleation and growth. If $\mathrm{d} \alpha / \mathrm{d} t$ increases with an increase in $\alpha$, the mechanism of desorption follows the contracting sphere model [11,37]. It can be seen from the $\alpha v s$. $\mathrm{d} \alpha / \mathrm{d} t$ curves (figure $6 \mathrm{c}$ ) that the mechanism of desorption for SC-SSS samples can be explained using the contracting sphere model while that of SC-NC follows the nucleation and growth model.

The change in mechanism can be understood by considering the available reaction sites in both the samples. Sample SC-NC having smaller particle and crystallite size might have a greater number of grain boundaries, where the oxygen surface exchange reaction could be faster. This indicates a greater number of nucleation sites for SC-NC sample compared to the SC-SSS sample, thus following nucleation and growth model of desorption. In contrast, SCSSS sample has bigger particles and thereby a lower number of active surface sites, which get nucleated fast covering the whole active surface with the reduced form $\left(\mathrm{SrCoO}_{2.5}\right)$. This apparently alters the reduction mechanism to contracting sphere model.

Further analysis of the desorption kinetics was performed by fitting the extent of conversion $v s$. time curve using a least square fitting method, assuming a first-order reaction. Only one exponential term was enough to fit the curve at all three temperatures for the SC-SSS sample, whereas two terms were required for the sample SC-NC. Two terms can be considered as related to oxygen surface exchange and oxide ion conduction in SC-NC whereas in SC-SSS sample, oxide ion conduction can be perceived as the rate limiting step. The fitting results are shown in table 3 and the red lines in figure $6 \mathrm{a}$ and $\mathrm{b}$ indicate the best fit to the data. Using the relaxation times $\left(\tau_{1}\right.$ and $\left.\tau_{2}\right)$ rate constants $\left(\mathrm{k}_{1}\right.$ and $\left.\mathrm{k}_{2}\right)$, respectively, were calculated. The rate constants follow an Arrhenius relation with temperature as shown in figure $6 \mathrm{~d}$. Activation energies calculated from the slope of curves are tabulated in table 3 .

\subsection{Electrical conductivity measurements}

The changes in oxygen non-stoichiometry of the oxide material with partial pressure of $\mathrm{O}_{2}$ changes the carrier concentration and thereby its electrical conductivity. Nonstoichiometric oxides can have electrons, holes as well as 
(a)

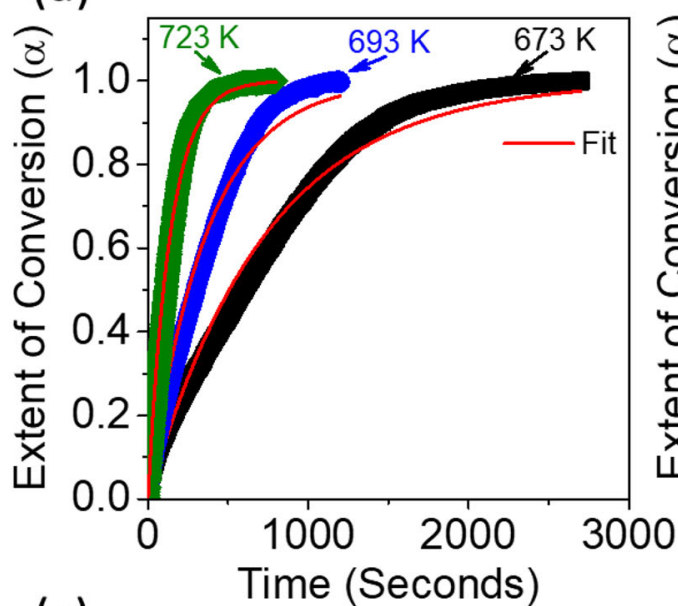

(c)

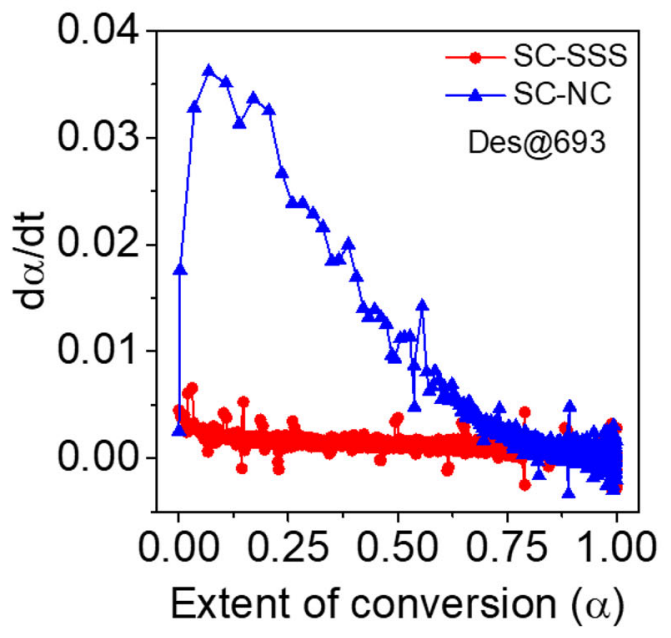

(b)

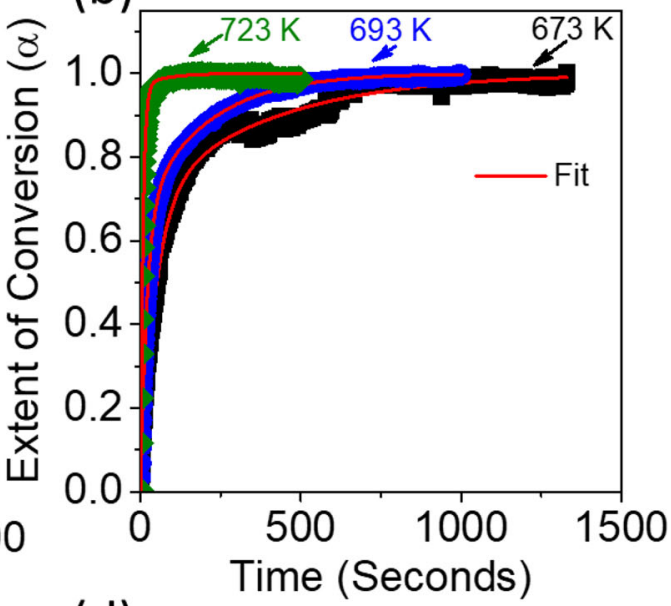

(d)

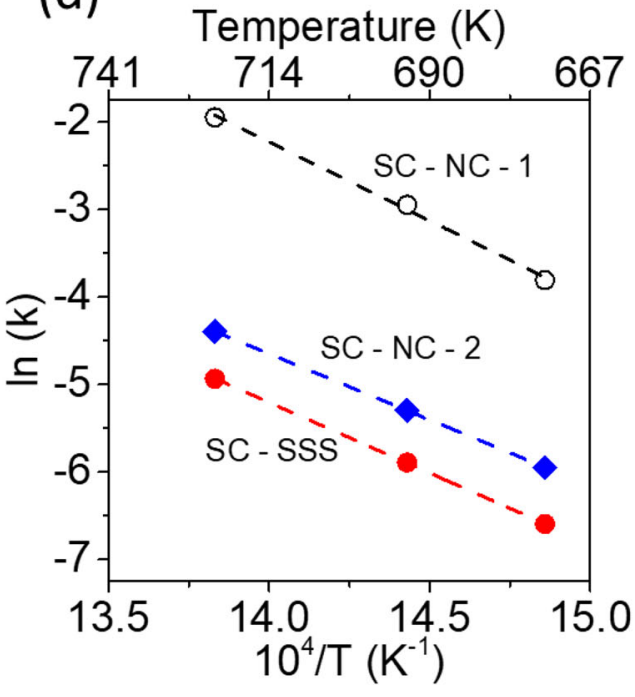

Figure 6. Time $v s$. the extent of conversion $(\alpha)$ curves for isothermal desorptions at 673,693 and $723 \mathrm{~K}$ for samples: (a) SC-SSS and (b) SC-NC. (c) $\alpha v s$. $\mathrm{d} \alpha / \mathrm{d} t$ for the samples SC-SSS and SC-NC desorbed at 693 K. (d) Arrhenius plots of rate constants for SC-SSS and SC-NC.

oxide ions as charge carries as per defect reactions in equations (1) and (2). Here Kröger-Vink notations, $\mathrm{V}_{\mathrm{O}}^{\bullet \bullet}, \mathrm{Co}_{\mathrm{Co}}^{x}, \mathrm{O}_{\mathrm{O}}^{x}, \mathrm{Co}_{\mathrm{Co}}^{\bullet}$ and $\mathrm{Co}_{\mathrm{Co}}^{\prime}$ denotes a vacancy of oxygen, $\mathrm{Co}^{3+}$ in a regular lattice site, an oxide ion in its lattice site, $\mathrm{Co}^{4+}$ in $\mathrm{Co}^{3+}$ lattice site (hole) and $\mathrm{Co}^{2+}$ in $\mathrm{Co}^{3+}$ lattice site (excess electrons), respectively. The excess electrons are produced by $\mathrm{Co}$ ion charge-disproportionation reaction (equation 2) at high temperatures and low oxygen partial pressures [38].

$$
\begin{aligned}
& \frac{1}{2} \mathrm{O}_{2}+\mathrm{V}_{\mathrm{o}}^{\bullet \bullet}+2 \mathrm{Co}_{\mathrm{Co}}^{x} \leftrightarrow \mathrm{O}_{\mathrm{O}}^{x}+2 \mathrm{Co}_{\mathrm{Co}}^{\bullet} \\
& 2 \mathrm{Co}_{\mathrm{Co}}^{x} \leftrightarrow \mathrm{Co}_{\mathrm{Co}}^{\bullet}+\mathrm{Co}_{\mathrm{Co}}^{\prime}
\end{aligned}
$$

The concentration of these charge carriers usually depends on the ambient oxygen partial pressure and temperature. Thus, the variation of electrical conductivity $(\sigma)$ with the temperature of SC-SSS and SC-NC samples were measured in the ambient air, flowing nitrogen and flowing oxygen atmospheres (figure 7a). Total conductivity increases with an increase in temperature indicating semiconducting behaviour for both SC-SSS and SC-NC samples in all three atmospheres. Both SC-SSS and SC-NC samples show similar behaviour in conductivity variation with temperature in the ambient air atmosphere. The room temperature electrical conductivity was found to be 0.01 and $0.02 \mathrm{~S} \mathrm{~cm}^{-1}$ for SC-SSS and SC-NC samples, respectively, in the ambient air atmosphere, which increases to a maximum value of 10 and $12 \mathrm{~S} \mathrm{~cm}^{-1}$ at $715 \mathrm{~K}$.

Conductivity in $\mathrm{N}_{2}$ as well as in $\mathrm{O}_{2}$ was found to be higher than that in the air for both the samples. Further, a sharp increase in conductivity at $\approx 600 \mathrm{~K}$ was observed for both the samples in all three atmospheres. The increase in conductivity in air and oxygen atmospheres might be due to 
Table 3. Fitting results for desorption kinetics study for SC-SSS and SC-NC samples.

\begin{tabular}{|c|c|c|c|c|c|c|c|}
\hline Desorption temperature $(\mathrm{K})$ & Equation & A1 & & $\tau_{1}(\mathrm{~s})$ & A2 & & $\tau_{2}(\mathrm{~s})$ \\
\hline \multicolumn{8}{|l|}{ SC-NC } \\
\hline 673 & $y=\mathrm{A} 1 \times \exp \left(-t / \tau_{1}\right)+\mathrm{A} 2 \times \exp \left(-t / \tau_{2}\right)+1$ & $-0.69(8)$ & & $45(1)$ & $-0.31(4)$ & & $383(5)$ \\
\hline 693 & & $-0.66(6)$ & & $19(1)$ & $-0.34(3)$ & & $199(1)$ \\
\hline 723 & & $-0.97(1)$ & & $7(1)$ & $-0.03(5)$ & & $81(15)$ \\
\hline Activation energy $(\mathrm{eV})$ & & & 1.55 & & & 1.30 & \\
\hline \multicolumn{8}{|l|}{ SC-SSS } \\
\hline 673 & $y=1-\exp \left(-t / \tau_{2}\right)$ & & & & & & $731(2)$ \\
\hline 693 & & & & & & & $362(2)$ \\
\hline 723 & & & & & & & $139(1)$ \\
\hline Activation energy $(\mathrm{eV})$ & & & & & & & 1.39 \\
\hline
\end{tabular}
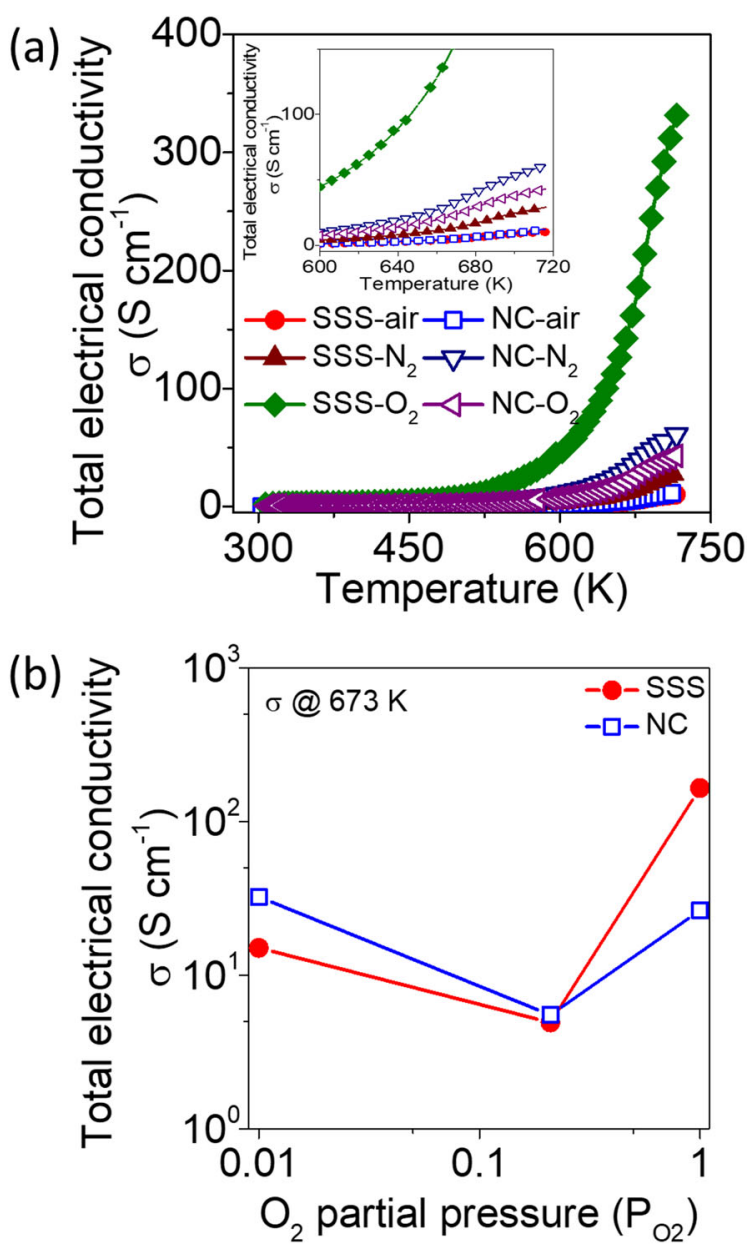

Figure 7. The electrical conductivity of the samples SC-SSS and SC-NC (a) in the ambient air, $\mathrm{N}_{2}$ and $\mathrm{O}_{2}$ atmospheres as a function of temperature and (b) as a function of partial pressure of $\mathrm{O}_{2}$ at $673 \mathrm{~K}$.

oxygen intake, whereby it increases both $p$-type as well as oxide ion conductivity. It should be noted that even though the total conductivity includes contribution from oxide ion conductivity, it might be negligible, of the order of

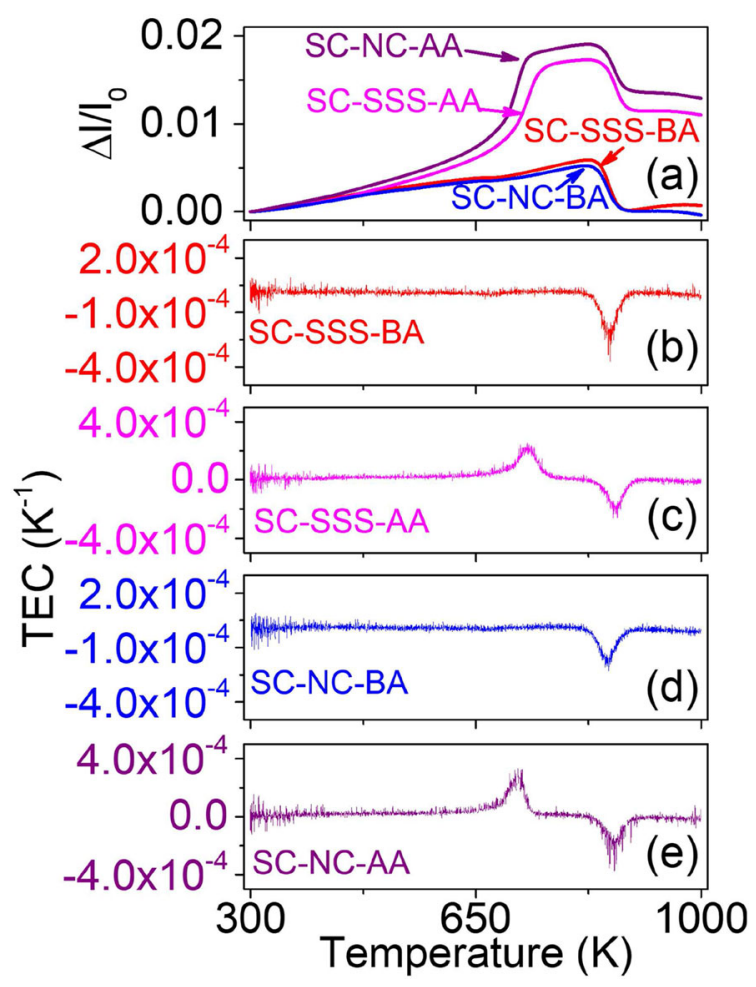

Figure 8. For the samples SC-SSS, SC-NC and those absorbed in 16 bar $\mathrm{O}_{2}$ at $673 \mathrm{~K}$ for $1 \mathrm{~h}$ : (a) linear thermal expansion $\left(\Delta l / l_{0}\right)$ and (b-e) variation of thermal expansion coefficients (TEC) with temperature. $\mathrm{BA}=$ before absorption, $\mathrm{AA}=$ after absorption.

$10^{-2} \mathrm{~S} \mathrm{~cm}^{-1}$ or less. In nitrogen atmosphere, reduction of the oxide and Co-ion disproportionation might be taking place above $600 \mathrm{~K}$ increasing the electron concentration and $n$-type conductivity.

Electrical conductivity of SC-SSS and SC-NC samples at $673 \mathrm{~K}$ (absorption temperature) in all three atmospheres were extracted from figure $7 \mathrm{a}$ and is plotted against the approximate oxygen partial pressure inside the measurement chamber (figure 7b). In $\mathrm{N}_{2}, \sigma$ was found to be higher for SC-NC than SC-SSS while in $\mathrm{O}_{2}, \sigma$ was higher for 
Table 4. Thermal expansion coefficients of the as-synthesized and absorbed SC-SSS and SC-NC samples.

\begin{tabular}{lccc}
\hline & \multicolumn{3}{c}{ Thermal expansion coefficient $\left(\mathrm{TEC} \times 10^{6} \mathrm{~K}^{-1}\right)$} \\
\cline { 2 - 4 } Sample code & As-synthesized $(T<750 \mathrm{~K})$ & Absorbed $(T<650 \mathrm{~K})$ & Absorbed $(750 \mathrm{~K}<T<830 \mathrm{~K})$ \\
\hline SC-SSS & 10.7 & 20.2 & 8.8 \\
SC-NC & 9.9 & 20.9 & 12.7 \\
\hline
\end{tabular}

\section{(a)}

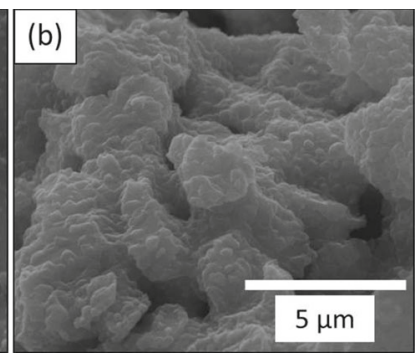

Figure 9. Scanning electron micrographs of (a) the SC-SSS and (b) the SC-NC samples after desorption.

SC-SSS than SC-NC. The same behaviour was observed at all other measured temperatures (300-715 K). Opposite behaviour of these samples' total electrical conductivity necessarily indicates a change in the conduction mechanism with the change in surrounding atmosphere, pointing towards a $p$ to $n$ transition with lowering oxygen partial pressure.

For cobalt-based microcrystalline ceramics of predominant electronic conductivity, increasing the grain size showed positive effects in ionic conductivity [16]. Similarly, in our case, the SC-SSS sample with higher crystallite and particle size showed a higher total electrical conductivity in the oxygen atmosphere than the SC-NC sample. This may be because of the higher ionic contribution to conductivity for SC-SSS samples above the absorption temperature due to its bigger particle size. In contrast, the electrical conductivity of the SC-NC sample was found to be higher than the SC-SSS sample in nitrogen atmosphere, may be due to a fast oxygen release as observed from the desorption kinetics study (section 3.4). Faster oxygen release indicates a greater number of electrons created increasing the total electrical conductivity.

\subsection{Thermal expansion measurements}

The oxygenated samples show a reduced unit cell volume compared to the as-synthesized samples (table 1 and table 2). This can give rise to a difference in thermal expansion between as-synthesized and oxygenated samples, which affects the reversible oxygen intake on cyclic oxidation/reduction treatments. Also, $\mathrm{BM} \quad \mathrm{SrCoO}_{2.5}$ synthesized through different methods may vary in their thermal expansion coefficients because of their differences in density. Hence, the linear expansion with temperature of the as-synthesized and absorbed samples were measured.

Linear thermal expansion $\left(\Delta l / l_{0}\right)$ and the variation of thermal expansion coefficients (TEC) with temperature of as-synthesized as well as absorbed SC-SSS and SC-NC samples are given in figure 8a-e. The thermal expansion behaviour of both the as-synthesized samples were observed to be similar. Thermal expansion coefficients are tabulated in table 4, showing no significant difference between the studied samples. Above $830 \mathrm{~K}$, a sharp change in expansion is observed for both the samples, where they get converted to the hexagonal phase of strontium cobaltite $\left(\mathrm{Sr}_{6} \mathrm{Co}_{5} \mathrm{O}_{15}\right)$. Oxygenated samples show a higher thermal expansion coefficient than as-synthesized samples, owing to the chemical expansion due to oxygen release. Further, the absorbed samples show a drastic change in expansion behaviour due to oxygen evolution above $650 \mathrm{~K}$. These samples as well get converted to hexagonal phase above $830 \mathrm{~K}$.

\subsection{Morphology after desorption}

The morphology of the samples after desorption is assessed for any changes after oxygen intake/release measurements (figure 9a and b). Small surface patches were seen on the surface of the SC-SSS and SC-NC samples after oxygen intake/release studies, which may be due to the formation of surface secondary phases $[11,39,40]$.

\section{Conclusions}

Effect of synthesis methods on the oxygen exchange kinetics of $\mathrm{BM} \mathrm{SrCoO}_{2.5}$ was explored. $\mathrm{BM} \mathrm{SrCoO}_{2.5}$ was synthesized through two different methods, solid-state reaction and nitrate-citrate gel method. Oxygen absorption/ desorption characteristics of these samples were analysed using a home-built volumetric set-up. Irrespective of the synthesis method used, the total oxygen holding capacity was found to be same for all three $\mathrm{BM} \mathrm{SrCoO}_{2.5}$ samples. The sample SC-SSS releases oxygen at a slower rate compared to SC-NC and follows contracting sphere model 
desorption, whereas SC-NC sample follows nucleation and growth kinetics for oxygen desorption.

\section{Acknowledgements}

We acknowledge Supercomputer Education and Research Centre (SERC), IISc, for the access of LabVIEW 7 program. AMN acknowledges Council of Scientific and Industrial Research (CSIR), Government of India, for the financial support in the form of Junior and Senior Research Fellowship. We also acknowledge FIST for providing funding for XRD, SEM and Dilatometry facilities.

\section{References}

[1] Fergus J W 2007 Sens. Actuators B Chem. 1231169

[2] Montini T, Melchionna M, Monai M and Fornasiero P 2016 Chem. Rev. 1165987

[3] Kim J-H and Manthiram A 2008 J. Electrochem. Soc. 155 B385

[4] Badwal S P S and Ciacchi F T 2001 Adv. Mater. 13993

[5] Geffroy P-M, Blond E, Richet N and Chartier T 2017 Chem. Eng. Sci. 162245

[6] Takeda Y, Kanno R, Takada T, Yamamoto O, Takano M and Bando Y 1986 Z. Anorg. Allg. Chem. 540259

[7] Rautama E-L and Karppinen M 2010 J. Solid State Chem. 1831102

[8] Klimkowicz A, Cichy K, Chmaissem O, Dabrowski B, Poudel B, Świerczek K et al 2019 J. Mater. Chem. A 72608

[9] Motohashi T, Ueda T, Masubuchi Y, Takiguchi M, Setoyama T, Oshima K et al 2010 Chem. Mater. 223192

[10] Dasgupta T, Sumithra S and Umarji A M 2008 Bull. Mater. Sci. 31859

[11] Narayanan A M and Umarji A M 2019 J. Alloys Compd. 803 102

[12] Lin Y S, MacLean D L and Zeng Y 2000 High temperature adsorption process, US Patent No. 6,059,858

[13] Wu H-C and Lin Y S 2017 Ind. Eng. Chem. Res. 566057

[14] Narayanan A M, Parasuraman R and Umarji A M 2018 Ind. Eng. Chem. Res. 5714749

[15] Jeen H, Choi W S, Biegalski M D, Folkman C M, Tung I-C, Fong D D et al 2013 Nat. Mater. 121057

[16] Reichmann M, Geffroy P-M, Richet N and Chartier T 2016 J. Power Sources 324774
[17] Guntuka S, Farooq S and Rajendran A 2008 Ind. Eng. Chem. Res. 47163

[18] Miura N, Ikeda H and Tsuchida A 2016 Ind. Eng. Chem. Res. 553091

[19] Ikeda H, Tsuchida A, Morita J and Miura N 2016 Ind. Eng. Chem. Res. 556501

[20] Beppu K, Hosokawa S, Shibano T, Demizu A, Kato K, Wada K et al 2017 Phys. Chem. Chem. Phys. 1914107

[21] Ding H, Xu Y, Luo C, Wang Q, Li S, Cai G et al 2017 Chem. Eng. J. 323340

[22] Nomura T, Zhu C, Sheng N, Murai R and Akiyama T $2015 \mathrm{~J}$. Alloys Compd. 646900

[23] West A R 2007 Solid state chemistry and its applications Student edn (New Delhi: Wiley India Pvt. Ltd.)

[24] Rao C N R and Gopalakrishnan J 1997 New directions in solid state chemistry 2nd edn (Cambridge, UK: Cambridge University Press)

[25] Jolivet J P, Henry M and Livage J 2000 Metal oxide chemistry and synthesis: from solution to solid state (Hoboken: Wiley-Blackwell)

[26] Lee B and Pope E J A 1994 Chemical processing of ceramics (Hoboken, USA: Wiley)

[27] Lee B and Komarneni S 2005 Chemical processing of ceramics 2nd edn (USA: CRC Press)

[28] Peláiz-Barranco A, Guerra J D S, López-Noda R and Araújo E B 2008 J. Phys. D: Appl. Phys. 41215503

[29] Patil K, Hegde M S, Rattan T and Aruna S T 2008 Chemistry of nanocrystalline oxide materials: combustion synthesis, properties and applications (Singapore: World Scientific)

[30] Bezdicka P, Wattiaux A, Grenier J, Pouchard M and Hagenmuller P 1993 Z. Anorg. Allg. Chem. 6197

[31] Wisgerhof E and Geus J W 1984 Mater. Res. Bull. 191591

[32] Toby B H and Von Dreele R B 2013 J. Appl. Crystallogr. 46 544

[33] Schneider C A, Rasband W S and Eliceiri K W 2012 Nat. Methods 9671

[34] van der Pauw L J 1958 Philips Res. Rep. 131

[35] Muñoz A, de la Calle C, Alonso J A, Botta P M, Pardo V, Baldomir D et al 2008 Phys. Rev. B 78054404

[36] Le Toquin R, Paulus W, Cousson A, Prestipino C and Lamberti C 2006 J. Am. Chem. Soc. 12813161

[37] Hurst N W, Gentry S J, Jones A and McNicol B D 1982 Catal. Rev. 24233

[38] Jin X, Wang J and Huang K 2017 Solid State Ionics 30948

[39] Crumlin E J, Mutoro E, Liu Z, Grass M E, Biegalski M D, Lee Y-L et al 2012 Energy Environ. Sci. 56081

[40] Hess F and Yildiz B 2020 Phys. Rev. Mater. 4015801 